\title{
Integrated Management of Alternaria Leaf Blight and Necrosis of Sunflower
}

\author{
P.N. Mane ${ }^{1}$, Manjusha S. Gaikwad ${ }^{2} *$, Prerna B. Chikte ${ }^{3}$ and Makarand P. Shinde ${ }^{4}$ \\ ${ }^{1}$ Oilseed Research Unit, ${ }^{2}$ College of Agriculture Gadchiroli, ${ }^{3}$ Pulses research Unit, \\ ${ }^{4}$ Agriculture Technology Information Center, Dr. PDKV., Akola, India
}

*Corresponding author

\section{A B S T R A C T}

\section{Keywords}

Oilseeds, Alternaria, necrosis, Trichoderma,

Pseudomonas

fluorescence,

Integrated

management

\section{Article Info}

Accepted:

26 February 2019

Available Online:

10 March 2019
Field experiment was conducted at Oilseeds Research Unit, Dr. Panjabrao Deshmukh Krishi Vidyapeeth, Akola during kharif 2013 -14 and 2015-16, to study integrated management of Alternaria leaf blight and necrosis of Sunflower. Seed primimg with Trichoderma viride@10g/kg seed, Carbendazim 50 WP @ 2g/kg seed + Thiamethoxam 600 FS @ 4g/kg seed, Trichoderma viride@10g/kg seed + Thiamethoxam 600 FS@ 4g/kg seed with foliar spray of Propiconazole 25 EC @ 1 ml/1 + Thiamethoxam 25 WG @ 1.6g/l, 25 EC @1 ml/1 + Azadirachtin 1500 ppm @ 1.5 ml/1, Pseudomonas fluorescence @ $5 \mathrm{~g} / \mathrm{l}+$ Azadirachtin 1500 ppm @ $1.5 \mathrm{ml} / 1$ were evaluated. Hydro priming has been used to infuse fungicides into seeds. Seed priming with Carbendazim 50 WP @ 2g/kg seed + Thiamethoxam 600 FS @ 4g/kg seed followed by spray of Propiconazole 25 EC @ 1ml/1 + Azadirachtin 1500 ppm @ $1.5 \mathrm{ml} / 1$ at onset of disease and 15 days later (T4) was found economical (1: 7.52 ICBR) and effective for the integrated management of Alternaria leaf blight and necrosis of Sunflower.

\section{Introduction}

Sunflower (Helianthus annuиs L.) is an important oil yielding crop, susceptible to a large variety of devastating diseases. Fungal diseases are the main constrain for sunflower industry Worldwide. Among the insect pest and diseases, diseases alone cause 10 per cent yield loss in the field itself. Alternaria blight is an important fungal disease of sunflower in India. It reduces the average flower size, number of seeds per plant, seed yield per plant, seed weight and per cent filling seed. Average yield and oil losses in India ranged from 28 to 80 per cent and 31 to 34 per cent, respectively (Balasubrahmanyam and Kolte, 1980). The disease is particularly destructive in regions where a combination of high temperature and extended periods of wet weather causes rapids epidemic development. Sunflower necrosis disease (SND) caused by tobacco streak virus has also emerged as a major disease of sunflower, First it was observed in Karnataka during the year 1997. Subsequently, it spread rapidly to other states with the disease incidence ranging from 2 90\% (Lavanya et al., 2009). In subsequent years, outbreaks of this disease in major 
sunflower-growing states of India, especially Andhra, Karanataka and Maharashtra, have virtually threatened the sunflower cultivation and yield losses ranging from 30 to $100 \%$ have been reported (Chander Rao et al., 2000). The disease was found to be transmitted by thrips (Harvir Singh, 2005). Researchers have focused in various aspects to manage the Alternaria and Necrosis. In the present study, integration of seed priming with biological and chemical fungicides, foliar spraying of chemical fungicide and botanical has been considered as a management approach.

\section{Materials and Methods}

Field experiment on integrated management of Alternaria leaf blight and necrosis of Sunflower was conducted at Oilseeds Research Unit, Dr. Panjabrao Deshmukh Krishi Vidyapeeth, Akola during kharif 2013 14 and 2015-16, to develop integrated module for foliar disease management in sunflower. The experiment was laid out in Randomized Block Design with seven treatments and three replications. Other recommended practices were followed and applied in all treatments. Sunflower seeds were pre soaked in water for $12 \mathrm{hrs}$ and treated with Trichoderma viride @ 10g/kg seed, Carbendazim 50 WP @ 2g/kg seed + Thiamethoxam 600 FS @ 4g/kg seed, Trichoderma viride @ $10 \mathrm{~g} / \mathrm{kg}$ seed + Thiamethoxam @ $4 \mathrm{~g} / \mathrm{kg}$. Foliar spray of Propiconazole 25 EC @ $1 \mathrm{ml} / \mathrm{l}+$ Thiamethoxam 25 WG @ $1.6 \mathrm{~g} / \mathrm{l}$, Propiconazole 25 EC @ 1ml/l + Azadirachtin 1500 ppm @ $1.5 \mathrm{ml} / 1$, Pseudomonas fluorescence@ 5 g/l + Azadirachtin 1500 ppm (a) $1.5 \mathrm{ml} / \mathrm{l}$ at disease onset, were evaluated. Hydro priming has been used to infuse fungicides into seeds. The treatment details were as given in table 1. Observations viz; per cent intensity of Alternaria leaf spots was recorded at one and two months after germination and necrosis was recorded at two months after germination.

\section{Results and Discussion}

\section{Seed Germination (\%)}

Statistically no significant difference was observed amongst the treatments towards germination of seed (Table 2).

\section{Per cent disease intensity of Alternaria leaf spot}

\section{One month after germination}

Two years pooled mean given in table 3 revealed that, minimum 15.87 per cent intensity of Alternaria leaf spot was recorded in treatment (T4) i.e seed bio priming with Carbendazim $50 \quad$ WP $@ 2 \mathrm{~g} / \mathrm{kg}$ seed + Thiamethoxam 600 FS @ 4g/kg seed \% + Spray of Propiconazole 25 EC @ 1ml/1 + Azadirachtin 1500 ppm @ $1.5 \mathrm{ml} / \mathrm{l}$ followed by T2 viz; seed biopriming with Carbendazim 50 WP @ 2g/kg seed + Thiamethoxam 600 FS @ 4g/kg seed + Two sprays of Propiconazole 25 EC@1ml/1 + Thiamethoxam25 WG @ 1.6 $\mathrm{g} / \mathrm{l}$ and T3 i.e Seed bio priming with $T$. viride @ $10 \mathrm{~g} / \mathrm{kg}$ seed + Two sprays of Propiconazole 25 EC@1ml/1 Azadirachtin 1500 ppm@ @ 1.5 $\mathrm{ml} / \mathrm{l}$ first at disease initiation and second at 15 days later showing 18.77 and 20.30 per cent intensity of Alternaria leaf spot respectively. All these treatments were statistically at par and significantly superior over rest of the treatments.

\section{Two month after germination}

Seed priming with Carbendazim 50 WP @ $2 \mathrm{~g} / \mathrm{kg}$ seed + Thiamethoxam 600 FS @ 4 g/kg seed + Two sprays of Propiconazole 25 EC @1ml/1 + Thiamethoxam 25 WG @ $1.6 \mathrm{~g} / \mathrm{l}$ at disease initiation and 15 days later (T2) recorded minimum i.e 17.43 per cent intensity of Alternaria leaf spot followed by seed priming with Carbendazim 50 WP @ 2g/kg seed + Thiamethoxam 600 FS @4g/kg seed + 
Two sprays of Propiconazole 25 EC @ 1ml/1 + Azadirachtin 1500 ppm@1.5 ml/l at disease onset and 15 days later (T4), Seed bio priming with T.viride@10g/kg seed + Two sprays of Propiconazole 25 EC @ 1ml/l + Azadirachtin 1500 ppm @1.5 ml/l at disease initiation and 15 days later (T3) and Seed biopriming with T. viride @ $10 \mathrm{~g} / \mathrm{kg}$ seed + Two sprays of Propiconazole $25 \quad$ EC @ $1 \mathrm{ml} / 1+$ Thiamethoxam $25 \mathrm{WG} @ 1.6 \mathrm{~g} / \mathrm{l}$ as soon as disease appeared and 15 days later (T1) recording 21.60, 21.83 and 21.87 per cent intensity of Alternaria leaf spot respectively, which were statistically at par with each other and were statistically superior over rest of the treatments including untreated control (Table $3)$.

\section{Necrosis}

Minimum incidence of necrosis 4.18 per cent was noticed due to treatment of seed priming with Carbendazim 50 WP @ 2g/kg seed + Thiamethoxam 600 FS @ 4g/kg seed + Two sprays of Propiconazole $25 \mathrm{EC} @ 1 \mathrm{ml} / 1+$ Azadirachtin 1500 ppm @ $1.5 \mathrm{ml} / 1$ as soon as disease appears and 15 days later (T4). Next best treatment with $4.54 \%$ disease incidence was seed bio priming with T. viride @ $10 \mathrm{~g} / \mathrm{kg}$ seed + Thiamethoxam @600 FS @ 4g/kg seed + Two sprays of Propiconazole 25 EC @1 ml/1 + Thiamethoxam 25 WG @ $1.6 \mathrm{~g} / \mathrm{l}$ as soon as disease appears and 15 days later (T6) followed by Seed priming with Carbendazim 50 WP @2g/kg seed + Thiamethoxam @600 FS @ 4g/kg seed + Two sprays of Propiconazole $25 \quad$ EC @ $1 \mathrm{ml} / 1+$ Thiamethoxam $25 \mathrm{WG} @ 1.6 \mathrm{~g} / \mathrm{l}$ as soon as disease appears and 15 days later (T2), Seed bio priming with $T$. viride @ $10 \mathrm{~g} / \mathrm{kg}$ seed + Two sprays of Propiconazole 25 EC @ 1 ml/1 + Azadirachtin 1500 ppm @ $1.5 \mathrm{ml} / 1$ as soon as disease appears and 15 days later (T3), Seed bio priming with $T$. viride @ $10 \mathrm{~g} / \mathrm{kg}$ seed + Two sprays of $P$. fluorescence @ $5 \mathrm{~g} / \mathrm{l}+$ Azadirachtin 1500 ppm @ $1.5 \mathrm{ml} / 1$ as soon as disease appears (T5) and Seed bio priming with T. viride@10g/ $\mathrm{kg}$ seed + Two sprays of Propiconazole 25 EC @1ml/1 + Thiamethoxam 25 WG @ $1.6 \mathrm{~g} / \mathrm{l}$ as soon as disease appears and 15 days later (T1) recording 4.96, 5.08, 5.16 and 5.91 per cent incidence of necrosis, respectively and were at par with each other (Table 4). However significantly superior over control (10.89\% incidence)

\section{Yield and economics}

Maximum yield of $1133 \mathrm{~kg} / \mathrm{ha}$ was obtained from Seed priming with Carbendazim 50 WP @ 2g/kg seed + Thiamethoxam 600 FS @ $4 \mathrm{~g} / \mathrm{kg}+$ Two sprays of Propiconazole 25 EC @1ml/1 + Thiamethoxam 25 WG @ $1.6 \mathrm{~g} / \mathrm{l}$ at disease appearance and 15 days later (T2) followed by Seed priming with Carbendazim 50 WP @ 2g/kg seed + Thiamethoxam 600 FS @ 4g/kg + Two sprays of Propiconazole 25 EC @1ml/1 + Azadirachtin 1500 ppm @ 1.5 $\mathrm{ml} / \mathrm{l}$ on disease onset and 15 days later (T4) and Seed bio priming with T. viride @ $10 \mathrm{~g} / \mathrm{kg}$ seed + Thiamethoxam 600 FS @ 4g/kg + Two sprays of Propiconazole 25 EC @ $1 \mathrm{ml} / 1+$ Thiamethoxam 25 WG@1.6 g/l at disease initiation and 15 days later (T6) recording 1100 and $1030 \mathrm{~kg} / \mathrm{ha}$, grain yield respectively. Al these three treatments were at par. However were significantly superior over control (Table 5).

Highest, 7.52 Incremental cost benefit was obtained from the treatment of seed priming with Carbendazim 50 WP @ 2g/kg seed + Thiamethoxam 600 FS @ 4g/kg + Two sprays of Propiconazole 25 EC @ $1 \mathrm{ml} / 1+$ Azadirachtin 1500 ppm @ $1.5 \mathrm{ml} / \mathrm{l}$ on disease appearance and 15 days later (T4) followed by seed bio priming with T. viride @ $10 \mathrm{~g} / \mathrm{kg}$ seed + Spray of P. fluorescence @ $5 \mathrm{~g} / 1+$ Azadirachtin 1500 ppm @ $1.5 \mathrm{ml} / \mathrm{l}$ as soon as disease appeared (T5), Seed priming with Carbendazim 50 WP @ $2 \mathrm{~g} / \mathrm{kg}$ seed + 
Thiamethoxam600 FS @ 4g/kg + Two sprays of Propiconazole $25 \quad \mathrm{EC} @ 1 \mathrm{ml} / \mathrm{l}+$ Thiamethoxam 25 WG @ $1.6 \mathrm{~g} / \mathrm{l}$ as soon as disease observed and 15 days later (T2), Seed bio priming with $T$. viride @ $10 \mathrm{~g} / \mathrm{kg}$ seed + Two sprays of Propiconazole 25 EC @ 1ml/l + Azadirachtin 1500 ppm @ $1.5 \mathrm{ml} / \mathrm{l}$ at disease initiation and 15 days later (T3), Seed bio priming with T. viride @ $10 \mathrm{~g} / \mathrm{kg}$ seed + Thiamethoxam 600 FS @ 4g/kg + Two sprays of Propiconazole $25 \quad \mathrm{EC} @ 1 \mathrm{ml} / \mathrm{l}+$ Thiamethoxam 25 WG @ $1.6 \mathrm{~g} / \mathrm{l}$ on disease appereance and 15 days later (T6) and Seed bio priming with $T$. viride @ $10 \mathrm{~g} / \mathrm{kg}$ seed + Two sprays of Propiconazole 25 EC @ 1ml/l + Thiamethoxam 25 WG @ $1.6 \mathrm{~g} / \mathrm{l}$ as soon as disease appeared and 15 days later (T1) (Table $5)$.

Results of the present study indicated that, seed bio priming with Carbendazim 50 WP @ 2g/kg seed + Thiamethoxam600 FS @ 4g/kg + Two sprays of Propiconazole 25 EC @ 1ml/1
+ Azadirachtin 1500 ppm @1.5 ml/l on disease initiation and 15 days later (T4) was effective and economical against Alternaria leaf spot and necrosis disease of sunflower.

Bio priming process had potential advantages over simple seed coating. Seed priming often results in more rapid and uniform seedling emergence and may be useful under adverse soil conditions (Mathre et al., 1994).

The infusion of fungicides into dormant seeds is a useful and efficient means of plant disease control. Hydro priming has been used to infuse systemic fungicides into seeds. The bacterial biocontrol agent may multiply substantially on seed during bio priming (Callan et al., 1990). Efficacy of Pseudomonas fluorescence as seed dresser can be enhanced by bio priming the sunflower seeds for the effective and eco-friendly management of Alternaria blight of sunflower.

Table.1 Treatment details

\begin{tabular}{|c|c|}
\hline S.N. & Treatments \\
\hline T1 & $\begin{array}{l}\text { Seed bio priming with Trichoderma viride @ } 10 \mathrm{~g} / \mathrm{kg} \text { seed + Two sprays of Propiconazole } \\
25 \mathrm{EC} @ 1 \mathrm{ml} / \mathrm{l}+\text { Thiamethoxam } 25 \mathrm{WG} @ 1.6 \mathrm{~g} / \mathrm{l} \text { as soon as disease appears and } 15 \text { days } \\
\text { later }\end{array}$ \\
\hline $\mathbf{T} 2$ & $\begin{array}{l}\text { Seed priming with Carbendazim } 50 \text { WP @ 2g/kg seed + Thiamethoxam } 600 \text { FS @ 4g/kg + } \\
\text { Two sprays of Propiconazole } 25 \text { EC @ 1ml/1 + Thiamethoxam } 25 \text { WG @ } 1.6 \mathrm{~g} / \mathrm{l} \text { as soon as } \\
\text { disease appears and } 15 \text { days later }\end{array}$ \\
\hline T3 & $\begin{array}{l}\text { Seed bio priming with Trichoderma viride @ } 10 \mathrm{~g} / \mathrm{kg} \text { seed + Two sprays of Propiconazole } \\
25 \mathrm{EC} @ 1 \mathrm{ml} / \mathrm{l}+\text { Azadirachtin } 1500 \mathrm{ppm} @ 1.5 \mathrm{ml} / 1 \text { as soon as disease appears and } 15 \\
\text { days later }\end{array}$ \\
\hline T4 & $\begin{array}{l}\text { Seed priming with Carbendazim } 50 \text { WP @ 2g/kg seed + Thiamethoxam } 600 \text { FS @ 4g/kg + } \\
\text { Two sprays of Propiconazole } 25 \text { EC @ 1ml/1 Azadirachtin } 1500 \text { ppm @ } 1.5 \mathrm{ml} / \mathrm{l} \text { as soon as } \\
\text { disease appears and } 15 \text { days later }\end{array}$ \\
\hline T5 & $\begin{array}{l}\text { Seed bio priming with Trichoderma viride @ } 10 \mathrm{~g} / \mathrm{kg} \text { seed + Two sprays of Pseudomonas } \\
\text { fluorescence @ } 5 \mathrm{~g} / \mathrm{l}+\text { Azadirachtin } 1500 \mathrm{ppm} @ 1.5 \mathrm{ml} / \mathrm{l} \text { as soon as disease appears. }\end{array}$ \\
\hline T6 & $\begin{array}{l}\text { Seed bio priming Trichoderma viride @ } 10 \mathrm{~g} / \mathrm{kg} \text { seed + Thiamethoxam } 600 \mathrm{FS} @ 4 \mathrm{~g} / \mathrm{kg}+ \\
\text { Two sprays of Propiconazole } 25 \mathrm{EC} @ 1 \mathrm{ml} / \mathrm{l}+\text { Thiamethoxam } 25 \mathrm{WG} @ 1.6 \mathrm{~g} / \mathrm{l} \text { as soon as } \\
\text { disease appears and } 15 \text { days later }\end{array}$ \\
\hline T7 & Control \\
\hline
\end{tabular}


Table.2 Effect of treatments on germination

\begin{tabular}{|c|c|c|c|c|}
\hline \multirow{2}{*}{\multicolumn{2}{|c|}{ Treatments }} & \multicolumn{3}{|c|}{ Seed germination (\%) } \\
\hline & & \multirow{2}{*}{$\begin{array}{c}2013-14 \\
97.31 \\
(9.86)^{*}\end{array}$} & \multirow{2}{*}{$\begin{array}{c}2015 \text { - } 16 \\
\\
77.46 \\
(8.80)^{*}\end{array}$} & \multirow{2}{*}{$\begin{array}{c}\begin{array}{c}\text { Pooled } \\
\text { mean }\end{array} \\
87.39 \\
(9.35)^{*}\end{array}$} \\
\hline 1 & $\begin{array}{l}\text { Seed bio priming with Trichoderma viride } \\
@ 10 \mathrm{~g} / \mathrm{kg} \text { seed }+ \text { Two sprays of Propiconazole } 25 \\
\text { EC @ } 1 \mathrm{ml} / \mathrm{l}+\text { Thiamethoxam } 25 \text { WG @ } 1.6 \mathrm{~g} / \mathrm{l} \text { as } \\
\text { soon as disease appears and } 15 \text { days later }\end{array}$ & & & \\
\hline 2 & $\begin{array}{l}\text { Seed priming with Carbendazim } 50 \text { WP @ 2g/kg } \\
\text { seed + Thiamethoxam 600 FS @ } 4 \mathrm{~g} / \mathrm{kg}+\text { Two } \\
\text { sprays of Propiconazole 25 EC @ } 1 \mathrm{ml} / 1+ \\
\text { Thiamethoxam 25 WG @ } 1.6 \mathrm{~g} / 1 \text { as soon as } \\
\text { disease appears and 15 days later }\end{array}$ & $\begin{array}{l}96.66 \\
(9.83)\end{array}$ & $\begin{array}{l}73.97 \\
(8.60)\end{array}$ & $\begin{array}{l}85.31 \\
(9.24)\end{array}$ \\
\hline 3 & $\begin{array}{l}\text { Seed bio priming with Trichoderma viride } \\
@ 10 \mathrm{~g} / \mathrm{kg} \text { seed + Two sprays of Propiconazole } 25 \\
\text { EC @ } 1 \mathrm{ml} / \mathrm{l}+\text { Azadirachtin } 1500 \mathrm{ppm} @ 1.5 \mathrm{ml} / 1 \\
\text { as soon as disease appears and } 15 \text { days later }\end{array}$ & $\begin{array}{l}97.14 \\
(9.86)\end{array}$ & $\begin{array}{l}73.34 \\
(8.56)\end{array}$ & $\begin{array}{l}85.24 \\
(9.23)\end{array}$ \\
\hline 4 & $\begin{array}{l}\text { Seed priming with Carbendazim } 50 \text { WP @ 2g/kg } \\
\text { seed + Thiamethoxam 600 FS @ 4g/kg + Two } \\
\text { sprays of Propiconazole 25 EC @ } 1 \mathrm{ml} / 1 \\
\text { Azadirachtin } 1500 \mathrm{ppm} @ 1.5 \mathrm{ml} / \mathrm{l} \text { as soon as } \\
\text { disease appears and } 15 \text { days later }\end{array}$ & $\begin{array}{l}97.14 \\
(9.86)\end{array}$ & $\begin{array}{l}77.78 \\
(8.82)\end{array}$ & $\begin{array}{l}87.46 \\
(9.35)\end{array}$ \\
\hline 5 & $\begin{array}{l}\text { Seed bio priming with Trichoderma viride } \\
@ 10 \mathrm{~g} / \mathrm{kg} \text { seed }+ \text { Two sprays of Pseudomonas } \\
\text { fluorescence @ } 5 \mathrm{~g} / \mathrm{l}+\text { Azadirachtin } 1500 \mathrm{ppm} @ \\
1.5 \mathrm{ml} / \mathrm{l} \text { as soon as disease appears. }\end{array}$ & $\begin{array}{l}96.97 \\
(9.85)\end{array}$ & $\begin{array}{l}73.33 \\
(8.56)\end{array}$ & $\begin{array}{l}85.15 \\
(9.23)\end{array}$ \\
\hline 6 & $\begin{array}{l}\text { Seed bio priming Trichoderma viride @ } 10 \mathrm{~g} / \mathrm{kg} \\
\text { seed + Thiamethoxam 600 FS @ } 4 \mathrm{~g} / \mathrm{kg}+\text { Two } \\
\text { sprays of Propiconazole 25 EC @ } 1 \mathrm{ml} / / \text { + } \\
\text { Thiamethoxam 25 WG @ } 1.6 \mathrm{~g} / 1 \text { as soon as } \\
\text { disease appears and 15 days later }\end{array}$ & $\begin{array}{l}97.61 \\
(9.88)\end{array}$ & $\begin{array}{l}78.41 \\
(8.86)\end{array}$ & $\begin{array}{l}88.01 \\
(9.38)\end{array}$ \\
\hline 7 & Control & $\begin{array}{l}97.93 \\
(9.90)\end{array}$ & $\begin{array}{l}67.94 \\
(8.24)\end{array}$ & $\begin{array}{l}82.93 \\
(9.11)\end{array}$ \\
\hline & ' $\mathrm{F}$ ' test & NS & NS & NS \\
\hline & $\mathrm{SE}(\mathrm{m}) \underline{ \pm}$ & - & - & - \\
\hline & $\mathrm{CD}$ at $5 \%$ & - & - & - \\
\hline & $\mathrm{CV} \%$ & - & - & - \\
\hline
\end{tabular}

\footnotetext{
* Square root transformed values.
} 
Table.3 Effect of treatments on intensity of Alternaria leaf spot

\begin{tabular}{|c|c|c|c|c|c|c|c|}
\hline \multirow{3}{*}{\multicolumn{2}{|c|}{ Treatments }} & \multicolumn{6}{|c|}{ Alternaria leaf spot ( \% intensity) } \\
\hline & & \multicolumn{3}{|c|}{ One month after germination } & \multicolumn{3}{|c|}{ Two month after germination } \\
\hline & & $2013-14$ & $2015-16$ & Pooled & $2013-14$ & $2015-16$ & $\begin{array}{c}\text { Pooled } \\
\text { mean }\end{array}$ \\
\hline 1 & $\begin{array}{l}\text { Seed bio priming with Trichoderma } \\
\text { viride @ } 10 \mathrm{~g} / \mathrm{kg} \text { seed }+ \text { Two sprays of } \\
\text { Propiconazole 25 EC @ } 1 \mathrm{ml} / 1+ \\
\text { Thiamethoxam } 25 \mathrm{WG} @ 1.6 \mathrm{~g} / \mathrm{l} \text { as soon } \\
\text { as disease appears and } 15 \text { days later }\end{array}$ & $\begin{array}{c}32.33 \\
(34.60)^{*}\end{array}$ & $\begin{array}{c}23.67 \\
(4.85)^{* *}\end{array}$ & $\begin{array}{c}28.00 \\
(31.89) \\
*\end{array}$ & $\begin{array}{c}27.00 \\
(31.28)^{*}\end{array}$ & $\begin{array}{c}16.73 \\
(19.34) *\end{array}$ & $\begin{array}{c}21.87 \\
(27.87)^{*}\end{array}$ \\
\hline 2 & $\begin{array}{l}\text { Seed priming with Carbendazim } 50 \mathrm{WP} \\
\text { @ } 2 \mathrm{~g} / \mathrm{kg} \text { seed }+ \text { Thiamethoxam } 600 \mathrm{FS} \\
\text { @ } 4 \mathrm{~g} / \mathrm{kg}+\text { Two sprays of Propiconazole } \\
25 \mathrm{EC} @ 1 \mathrm{ml} / \mathrm{l}+\text { Thiamethoxam } 25 \mathrm{WG} \\
@ 1.6 \mathrm{~g} / \mathrm{l} \text { as soon as disease appears and } \\
15 \text { days later }\end{array}$ & $\begin{array}{c}23.87 \\
(29.18)\end{array}$ & $\begin{array}{l}13.67 \\
(3.66)\end{array}$ & $\begin{array}{c}18.77 \\
(25.57)\end{array}$ & $\begin{array}{l}20.13 \\
(26.64)\end{array}$ & $\begin{array}{c}14.80 \\
(16.05)\end{array}$ & $\begin{array}{c}17.43 \\
(24.65)\end{array}$ \\
\hline 3 & $\begin{array}{l}\text { Seed bio priming with Trichoderma } \\
\text { viride @ } 10 \mathrm{~g} / \mathrm{kg} \text { seed }+ \text { Two sprays of } \\
\text { Propiconazole } 25 \mathrm{EC} @ 1 \mathrm{ml} / 1+ \\
\text { Azadirachtin } 1500 \mathrm{pm} @ 1.5 \mathrm{ml} / 1 \text { as } \\
\text { soon as disease appears and } 15 \text { days later }\end{array}$ & $\begin{array}{c}24.33 \\
(29.49)\end{array}$ & $\begin{array}{l}16.27 \\
(4.03)\end{array}$ & $\begin{array}{c}20.30 \\
(26.74)\end{array}$ & $\begin{array}{l}26.67 \\
(31.03)\end{array}$ & $\begin{array}{c}17.00 \\
(19.54)\end{array}$ & $\begin{array}{c}21.83 \\
(27.81)\end{array}$ \\
\hline 4 & $\begin{array}{l}\text { Seed priming with Carbendazim 50 WP } \\
\text { @ } 2 \mathrm{~g} / \mathrm{kg} \text { seed }+ \text { Thiamethoxam 600 FS } \\
\text { @ } 4 \mathrm{~g} / \mathrm{kg}+\text { Two sprays of Propiconazole } \\
25 \mathrm{EC} @ 1 \mathrm{ml} / 1 \text { Azadirachtin } 1500 \mathrm{ppm} \\
@ 1.5 \mathrm{ml} / \mathrm{l} \text { as soon as disease appears } \\
\text { and } 15 \text { days later }\end{array}$ & $\begin{array}{c}19.13 \\
(25.92)\end{array}$ & $\begin{array}{l}12.60 \\
(3.55)\end{array}$ & $\begin{array}{c}15.87 \\
(23.46)\end{array}$ & $\begin{array}{c}30.20 \\
(33.33)\end{array}$ & $\begin{array}{c}13.07 \\
(16.51)\end{array}$ & $\begin{array}{c}21.60 \\
(27.69)\end{array}$ \\
\hline 5 & $\begin{array}{l}\text { Seed bio priming with Trichoderma } \\
\text { viride @ } 10 \mathrm{~g} / \mathrm{kg} \text { seed }+ \text { Two sprays of } \\
\text { Pseudomonas fluorescence @ } 5 \mathrm{~g} / \mathrm{l}+ \\
\text { Azadirachtin } 1500 \mathrm{ppm} @ 1.5 \mathrm{ml} / 1 \text { as } \\
\text { soon as disease appears. }\end{array}$ & $\begin{array}{c}48.00 \\
(43.85)\end{array}$ & $\begin{array}{l}22.20 \\
(4.71)\end{array}$ & $\begin{array}{c}35.10 \\
(36.31)\end{array}$ & $\begin{array}{c}49.33 \\
(44.61)\end{array}$ & $\begin{array}{c}22.73 \\
(24.50)\end{array}$ & $\begin{array}{c}36.00 \\
(36.84)\end{array}$ \\
\hline 6 & $\begin{array}{l}\text { Seed bio priming Trichoderma viride } \\
\text { @ } 10 \mathrm{~g} / \mathrm{kg} \text { seed }+ \text { Thiamethoxam } 600 \mathrm{FS} \\
\text { @ } 4 \mathrm{~g} / \mathrm{kg}+\text { Two sprays of Propiconazole } \\
25 \mathrm{EC} @ 1 \mathrm{ml} / 1+\text { Thiamethoxam } 25 \mathrm{WG} \\
@ 1.6 \mathrm{~g} / \mathrm{l} \text { as soon as disease appears and } \\
15 \text { days later }\end{array}$ & $\begin{array}{c}57.93 \\
(49.57)\end{array}$ & $\begin{array}{l}18.27 \\
(5.70)\end{array}$ & $\begin{array}{c}38.10 \\
(38.11)\end{array}$ & $\begin{array}{c}59.20 \\
(50.31)\end{array}$ & $\begin{array}{c}27.67 \\
(25.99)\end{array}$ & $\begin{array}{c}43.40 \\
(38.15)\end{array}$ \\
\hline 7 & Control & $\begin{array}{c}60.07 \\
(50.86)\end{array}$ & $\begin{array}{l}27.53 \\
(5.24)\end{array}$ & $\begin{array}{c}43.80 \\
(41.42)\end{array}$ & $\begin{array}{c}62.13 \\
(52.09)\end{array}$ & $\begin{array}{c}30.67 \\
(33.59)\end{array}$ & $\begin{array}{c}45.37 \\
(42.33)\end{array}$ \\
\hline & 'F' test & Sig & Sig & Sig & Sig & Sig & Sig \\
\hline & $\mathrm{SE}(\mathrm{m}) \pm$ & 7.73 & 0.20 & 1.53 & 6.77 & 1.22 & 1.22 \\
\hline & $\mathrm{CD}$ at $5 \%$ & 8.57 & 0.61 & 4.73 & 8.02 & 3.77 & 3.76 \\
\hline & $\mathrm{CV} \%$ & 12.11 & 7.67 & 8.32 & 11.04 & 7.98 & 6.47 \\
\hline
\end{tabular}

* Arc sine transformed values

$* *$ Square root transformed values 
Table.4 Effect of treatments on incidence of necrosis

\begin{tabular}{|c|c|c|c|c|}
\hline \multirow{2}{*}{\multicolumn{2}{|c|}{ Treatments }} & \multicolumn{3}{|c|}{$\begin{array}{c}\text { Necrosis }(\%) \\
\text { Two month after germination }\end{array}$} \\
\hline & & \multirow{2}{*}{$\begin{array}{c}2013-14 \\
0.98 \\
(1.16)\end{array}$} & \multirow{2}{*}{$\begin{array}{c}2015-16 \\
10.83 \\
(3.25)\end{array}$} & \multirow{2}{*}{\begin{tabular}{c|}
$\begin{array}{c}\text { Pooled } \\
\text { mean }\end{array}$ \\
5.91 \\
$(2.41)$
\end{tabular}} \\
\hline 1 & $\begin{array}{l}\text { Seed bio priming with Trichoderma viride @ } 10 \mathrm{~g} / \mathrm{kg} \text { seed } \\
+ \text { Two sprays of Propiconazole } 25 \mathrm{EC} 1 \mathrm{ml} / \mathrm{l}+ \\
\text { Thiamethoxam 25 WG @ } 1.6 \mathrm{~g} / \mathrm{l} \text { as soon as disease } \\
\text { appears and } 15 \text { days later }\end{array}$ & & & \\
\hline 2 & $\begin{array}{l}\text { Seed priming with Carbendazim } 50 \text { WP @ 2g/kg seed + } \\
\text { Thiamethoxam 600 FS @ 4g/kg + Two sprays of } \\
\text { Propiconazole 25 EC @1ml/ + Thiamethoxam 25 WG @ } \\
1.6 \mathrm{~g} / \mathrm{l} \text { as soon as disease appears and } 15 \text { days later }\end{array}$ & $\begin{array}{c}0.00 \\
(0.71)\end{array}$ & $\begin{array}{c}9.92 \\
(3.11)\end{array}$ & $\begin{array}{c}4.96 \\
(2.20)\end{array}$ \\
\hline 3 & $\begin{array}{l}\text { Seed bio priming with Trichoderma viride @ } 10 \mathrm{~g} / \mathrm{kg} \text { seed } \\
+ \text { Two sprays of Propiconazole } 25 \mathrm{EC} 1 \mathrm{ml} / \mathrm{l}+ \\
\text { Azadirachtin } 1500 \mathrm{ppm} @ 1.5 \mathrm{ml} / 1 \text { as soon as disease } \\
\text { appears and } 15 \text { days later }\end{array}$ & $\begin{array}{c}0.64 \\
(1.04)\end{array}$ & $\begin{array}{c}9.51 \\
(3.08)\end{array}$ & $\begin{array}{l}5.08 \\
(2.24)\end{array}$ \\
\hline 4 & $\begin{array}{l}\text { Seed priming with Carbendazim } 50 \mathrm{WP} @ 2 \mathrm{~g} / \mathrm{kg} \text { seed }+ \\
\text { Thiamethoxam 600 FS @ 4g/kg + Two sprays of } \\
\text { Propiconazole } 25 \mathrm{EC} @ 1 \mathrm{ml} / \mathrm{l} \text { Azadirachtin } 1500 \mathrm{ppm} @ \\
1.5 \mathrm{ml} / \mathrm{l} \text { as soon as disease appears and } 15 \text { days later }\end{array}$ & $\begin{array}{c}0.99 \\
(1.22)\end{array}$ & $\begin{array}{c}7.38 \\
(2.70)\end{array}$ & $\begin{array}{c}4.18 \\
(2.03)\end{array}$ \\
\hline 5 & $\begin{array}{l}\text { Seed bio priming with Trichoderma viride @ } 10 \mathrm{~g} / \mathrm{kg} \text { seed } \\
+ \text { Two sprays of Pseudomonas fluorescence @ } 5 \mathrm{~g} / \mathrm{l}+ \\
\text { Azadirachtin } 1500 \mathrm{ppm} @ 1.5 \mathrm{ml} / \mathrm{l} \text { as soon as disease } \\
\text { appears. }\end{array}$ & $\begin{array}{c}0.32 \\
(0.87)\end{array}$ & $\begin{array}{l}10.00 \\
(3.16)\end{array}$ & $\begin{array}{c}5.16 \\
(2.27)\end{array}$ \\
\hline 6 & $\begin{array}{l}\text { Seed bio priming Trichoderma viride @ } 10 \mathrm{~g} / \mathrm{kg} \text { seed + } \\
\text { Thiamethoxam600 FS @ 4g/kg + Two sprays of } \\
\text { Propiconazole 25 EC @ } 1 \mathrm{ml} / \mathrm{l}+\text { Thiamethoxam 25 WG @ } \\
1.6 \mathrm{~g} / \mathrm{l} \text { as soon as disease appears and } 15 \text { days later }\end{array}$ & $\begin{array}{c}0.97 \\
(1.16)\end{array}$ & $\begin{array}{c}8.11 \\
(2.76)\end{array}$ & $\begin{array}{c}4.54 \\
(2.05)\end{array}$ \\
\hline \multirow[t]{5}{*}{7} & Control & $\begin{array}{c}6.48 \\
(2.64)\end{array}$ & $\begin{array}{l}15.29 \\
(3.88)\end{array}$ & $\begin{array}{l}10.89 \\
(3.28)\end{array}$ \\
\hline & 'F' test & Sig & Sig & Sig \\
\hline & $\mathrm{SE}(\mathrm{m}) \underline{ \pm}$ & 0.17 & 0.33 & 0.25 \\
\hline & $\mathrm{CD}$ at $5 \%$ & 0.53 & 1.02 & 0.76 \\
\hline & $\mathrm{CV} \%$ & 23.81 & 18.27 & 18.05 \\
\hline
\end{tabular}

* Figures in parenthesis are square root transformed values 
Table.5 Yield and ICBR

\begin{tabular}{|c|c|c|c|c|c|}
\hline \multirow{2}{*}{\multicolumn{2}{|c|}{ Treatments }} & \multicolumn{3}{|c|}{ Seed yield $\mathrm{kg} / \mathrm{ha}$} & \multirow{3}{*}{\begin{tabular}{|l|} 
ICBR \\
2.98 \\
\end{tabular}} \\
\hline & & \multirow{2}{*}{$\begin{array}{c}2013-14 \\
640\end{array}$} & \multirow{2}{*}{$\begin{array}{c}2015-16 \\
1165\end{array}$} & \multirow{2}{*}{$\begin{array}{c}\begin{array}{c}\text { Pooled } \\
\text { mean }\end{array} \\
902 \\
\end{array}$} & \\
\hline 1 & $\begin{array}{l}\text { Seed bio priming with Trichoderma viride @ } 10 \mathrm{~g} / \mathrm{kg} \text { seed } \\
+ \text { Two sprays of Propiconazole 25 EC @ } 1 \mathrm{ml} / 1+ \\
\text { Thiamethoxam 25 WG @ } 1.6 \mathrm{~g} / \mathrm{l} \text { as soon as disease } \\
\text { appears and } 15 \text { days later }\end{array}$ & & & & \\
\hline 2 & $\begin{array}{l}\text { Seed priming with Carbendazim 50 WP @ 2g/kg seed + } \\
\text { Thiamethoxam 600 FS @ 4g/kg + Two sprays of } \\
\text { Propiconazole 25 EC @ 1ml/1 + Thiamethoxam 25 WG @ } \\
1.6 \mathrm{~g} / \mathrm{l} \text { as soon as disease appears and } 15 \text { days later }\end{array}$ & 746 & 1519 & 1133 & 4.62 \\
\hline 3 & $\begin{array}{l}\text { Seed bio priming with Trichoderma viride @ } 10 \mathrm{~g} / \mathrm{kg} \text { seed } \\
+ \text { Two sprays of Propiconazole 25 EC @ } 1 \mathrm{ml} / \mathrm{l}+ \\
\text { Azadirachtin } 1500 \mathrm{ppm} @ 1.5 \mathrm{ml} / \mathrm{l} \text { as soon as disease } \\
\text { appears and } 15 \text { days later }\end{array}$ & 664 & 1053 & 858 & 4.32 \\
\hline 4 & $\begin{array}{l}\text { Seed priming with Carbendazim } 50 \text { WP @ 2g/kg seed + } \\
\text { Thiamethoxam 600 FS @ 4g/kg + Two sprays of } \\
\text { Propiconazole 25 EC @ } 1 \mathrm{ml} / 1 \text { Azadirachtin } 1500 \mathrm{ppm} @ \\
1.5 \mathrm{ml} / \mathrm{l} \text { as soon as disease appears and } 15 \text { days later }\end{array}$ & 618 & 1582 & 1100 & 7.52 \\
\hline 5 & $\begin{array}{l}\text { Seed bio priming with Trichoderma viride @ } 10 \mathrm{~g} / \mathrm{kg} \text { seed } \\
+\quad \text { Two sprays of Pseudomonas fluorescence @ } 5 \mathrm{~g} / \mathrm{l}+ \\
\text { Azadirachtin } 1500 \mathrm{ppm} @ 1.5 \mathrm{ml} / \mathrm{l} \text { as soon as disease } \\
\text { appears. }\end{array}$ & 585 & 1171 & 878 & 5.00 \\
\hline 6 & $\begin{array}{l}\text { Seed bio priming Trichoderma viride @ } 10 \mathrm{~g} / \mathrm{kg} \text { seed + } \\
\text { Thiamethoxam 600 FS @ 4g/kg + Two sprays of } \\
\text { Propiconazole 25 EC @ } 1 \mathrm{ml} / 1+\text { Thiamethoxam 25 WG @ } \\
1.6 \mathrm{~g} / \mathrm{l} \text { as soon as disease appears and } 15 \text { days later }\end{array}$ & 557 & 1502 & 1030 & 3.67 \\
\hline \multirow[t]{5}{*}{7} & Control & 304 & 836 & 570 & - \\
\hline & ' $\mathrm{F}$ ' test & Sig & $\mathrm{Sig}$ & Sig & - \\
\hline & $\mathrm{SE}(\mathrm{m}) \underline{ \pm}$ & 38.41 & 84.50 & 45.75 & - \\
\hline & $\mathrm{CD}$ at $5 \%$ & 118.34 & 260.38 & 140.97 & - \\
\hline & $\mathrm{CV} \%$ & 11.32 & 11.61 & 8.57 & - \\
\hline
\end{tabular}

The effectiveness of carbendazim in controlling blight of sunflower caused by $A$. helianthi, with increased yield was reported by Patel et al., (1995).
Sunflower necrosis disease can be managed by treating seeds with thiomethoxam at $4 \mathrm{~g} / \mathrm{kg}$ along with two sprays of the chemical at $0.05 \%$ at $30 \& 45$ DAS (Shirshikar, 2010). 
The incidence of sunflower necrosis disease can be minimized by seed treatment with imidacloprid 70 W.S., @ 5 g/kg (Shirshikar, 2008).

\section{References}

Anonymous. 2002. Research Highlights 1997-2000. Directorate of Oilseeds Research, Hyderbad.: 70-75.

Balasubrahmanyam, N and S. J. Kolte. 1980. Effect of Alternaria blight on yield components, oil content and seed quality of sunflower. Indian J. Agric. Sci. 50(9): 701-706

Callan, N.W., D.E. Mathre and J.B Miller. 1990. Bio-priming seed treatment for biological control of Pythium ultimum pre emergence damping off in sh2 sweet corn. Plant Disease. 74: 368372.

Chander, Rao. S., M.A. Raoof and H. Singh. 2000. Sunflower necrosis disease, a preliminary study on transmission. In: Proceedings of National Seminar on Oilseeds and Oilseed Research Development Needs in the 3rd Millennium. Indian Soc. of Oilseeds Res. (DOR), Hyderabad, during Feb. 2-4.: 285-286.

Chattopadhyay C. 1999. Yield loss attributable to Alternaria blight of sunflower (Helianthus annuus L.) in India and some potentially effective control measures. International journal of pest management. 45(1) 15-21.
Harvir Singh. 2005. Thrips incidence and necrosis disease in sunflower. $J$. Oilseeds Res. 22 (1): 90-92.

Lavanya, N., D. Saravanakumar, L. Rajendran, M. Ramiah, T. Raguchander and R. Samiyappan. 2009. Management of sunflower necrosis virus through antiviral substances. Archives of Phytopathology and Plant Protection. 42(3): 265-276.

Mathre, D.E., N.W. Callan and A. Schwend. 1994. Factors influencing the control of Pythium ultimum-induced seed decay by seed treatment with Pseudomonas aureofaciens AB254. Crop Protection. 13: 301-307.

Patel, V. A., K. A. Vaishnav and B. K. Kikari. 1995. Fungicidal management of leaf spot (Alternaria helianthi) of sunflower. Indian Journal of Mycology and Plant Pathology. 25: 113.

Rao, M.S.L, S. Kulkarni., S. Lingaraju and H.L. Nadaf. 2009. Bio-priming of seeds: A potential tool in the integrated management of Alternaria blight of Sunflower. Helia, 32 (50): 107-114

Shirshikar, S.P. 2008. Integrated Management of Sunflower Necrosis Disease. Helia. 31(49): 27-34

Shirshikar, S.P. 2010. Sunflower Necrosis Disease Management with Thiomethoxam. Helia. 33(53): 63-68.

\section{How to cite this article:}

Mane, P.N., Manjusha S. Gaikwad, Prerna B. Chikte and Makarand P. Shinde. 2019. Integrated Management of Alternaria Leaf Blight and Necrosis of Sunflower. Int.J.Curr.Microbiol.App.Sci. 8(03): 2536-2544. doi: https://doi.org/10.20546/ijcmas.2019.803.301 